\title{
Determinantal construction of orthogonal polynomials associated with root systems
}

\author{
J. F. van Diejen, L. Lapointe and J. Morse
}

\begin{abstract}
We present an explicit construction of the Macdonald polynomials and of the HeckmanOpdam generalized Jacobi polynomials associated with root systems.
\end{abstract}

\section{Introduction}

The main objective of this work concerns the explicit computation of families of orthogonal polynomials associated with root systems. Key examples of the families under consideration are the Macdonald polynomials [Mac95, Mac98, Mac01] and the Heckman-Opdam generalized Jacobi polynomials [HS94, Opd95]. The origin of the Heckman-Opdam polynomials lies in the harmonic analysis of simple Lie groups, where they appear (for special parameter values) as zonal spherical functions on compact symmetric spaces [HS94, Hel94]. Other important applications of these polynomials arise in mathematical physics, where they are used to express the eigenfunctions of the quantum Calogero-Sutherland one-dimensional many-body systems [Sut71, Sut72, OP83, HS94]. The Macdonald polynomials have similar applications: they appear as zonal spherical functions on compact quantum symmetric spaces [Nou96, Sug99], and they are used to express the eigenfunctions of Ruijsenaars' $(q$-)difference Calogero-Sutherland systems [Rui87, Die96]. Depending on the specific application of interest, our work may thus be viewed as providing an explicit construction for the zonal spherical functions on compact (quantum) symmetric spaces or for the eigenfunctions of the (difference) Calogero-Sutherland type quantum many-body models.

The usual definition of the Heckman-Opdam and Macdonald polynomials involves a GramSchmidt type orthogonalization of the monomial basis with respect to a generalized Haar measure [HS94, Opd95, Mac98, Mac01]. This definition, although most appropriate from a theoretical point of view, is not very adequate for the explicit computation of the polynomials in question. The main result of this paper is a determinantal formula for the Heckman-Opdam and Macdonald polynomials that gives rise to an efficient recursive procedure from which their expansion in the monomial basis can be constructed explicitly. For the type $A$ root systems the Heckman-Opdam polynomials reduce (in essence) to Jack's polynomials [Sta89, Mac95] and the Macdonald polynomials reduce to Macdonald's symmetric functions [Mac95]. In this case the determinantal construction of the polynomials under consideration was laid out in previous work by Lapointe, Lascoux and Morse [LLM98, LLM00]. More specifically, the results of the present paper constitute a generalization of the methods of [LLM98, LLM00] to the case of arbitrary root systems. For the Heckman-Opdam families we consider general (not necessarily reduced) root systems and general values of the root multiplicity parameters. For the Macdonald families, however, we restrict for technical reasons to

Received 28 March 2002, accepted in final form 7 March 2003.

2000 Mathematics Subject Classification 05E35 (primary), 33C52 (secondary).

Keywords: Macdonald polynomials, Heckman-Opdam, polynomials, determinantal formulas, root systems.

Work supported in part by the Fondo Nacional de Desarrollo Científico y Tecnológico (FONDECYT) Grants \# 1010217 and \# 7010217, the Programa Formas Cuadráticas of the Universidad de Talca, the Cátedra Presidencial in Number Theory, and NSF Grant \# 0100179.

This journal is (C) Foundation Compositio Mathematica 2004. 


\section{J. F. van Diejen, L. Lapointe and J. Morse}

those (reduced) root systems for which the dual root system $R^{\vee}$ has a minuscule weight (thus including the types $A_{N}, B_{N}, C_{N}, D_{N}, E_{6}$ and $E_{7}$ while excluding the types $B C_{n}, E_{8}, F_{4}$ and $G_{2}$ ).

\section{Triangular operators in the symmetrized group algebra}

In this section we define the concept of a triangular operator in the Weyl-group invariant component of the group algebra over the weight lattice of a root system. For preliminaries on root systems the reader is referred to e.g. [Bou68, Hum72].

Let $E,\langle\cdot, \cdot\rangle$ be a real Euclidean space spanned by an irreducible root system $R$ with Weyl group $W$. We write $\mathcal{Q}$ and $\mathcal{Q}^{+}$for the root lattice and its non-negative semigroup generated by the positive roots $R^{+}$

$$
\mathcal{Q}=\operatorname{Span}_{\mathbb{Z}}(R), \quad \mathcal{Q}^{+}=\operatorname{Span}_{\mathbb{N}}\left(R^{+}\right) .
$$

The weight lattice $\mathcal{P}$ and the cone of dominant weights $\mathcal{P}^{+}$are given by

$$
\mathcal{P}=\left\{\lambda \in E \mid\left\langle\lambda, \alpha^{\vee}\right\rangle \in \mathbb{Z}, \forall \alpha \in R\right\}
$$

and

$$
\mathcal{P}^{+}=\left\{\lambda \in E \mid\left\langle\lambda, \alpha^{\vee}\right\rangle \in \mathbb{N}, \forall \alpha \in R^{+}\right\},
$$

where $\alpha^{\vee}=2 \alpha /\langle\alpha, \alpha\rangle$. The weight lattice is endowed with the natural partial order

$$
\lambda \succeq \mu \Longleftrightarrow \lambda-\mu \in \mathcal{Q}^{+} .
$$

Let $\mathcal{Q}^{\vee}$ denote the dual root lattice generated by the dual root system $R^{\vee}=\left\{\alpha^{\vee} \mid \alpha \in R\right\}$. The group algebra over the weight lattice $\mathbb{R}[\mathcal{P}]$ is the algebra generated by the formal exponentials $e^{\lambda}, \lambda \in \mathcal{P}$ subject to the multiplication relation $e^{\lambda} e^{\mu}=e^{\lambda+\mu}$. This algebra can be realized explicitly as the algebra $\mathcal{A}$ of (Fourier) polynomials on the torus $\mathbb{T}=E /\left(2 \pi \mathcal{Q}^{\vee}\right)$ through the identification

$$
e^{\lambda}=e^{i\langle\lambda, x\rangle}, \quad \lambda \in \mathcal{P}
$$

(with $x \in \mathbb{T}$ ). Symmetrization with respect to the action of the Weyl group produces the basis of monomial symmetric functions $\left\{m_{\lambda}\right\}_{\lambda \in \mathcal{P}+}$ for the space $\mathcal{A}^{W}$ of Weyl-group invariant polynomials on $\mathbb{T}$, where

$$
m_{\lambda}=\sum_{\mu \in W(\lambda)} e^{\mu}, \quad \lambda \in \mathcal{P},
$$

with $W(\lambda)$ denoting the orbit of $\lambda$ with respect to the action of the Weyl group.

We write $\mathcal{A}_{\lambda}^{W}$ for the finite-dimensional highest weight subspace of $\mathcal{A}^{W}$ with highest weight $\lambda \in \mathcal{P}^{+}$, i.e., $\mathcal{A}_{\lambda}^{W}=\operatorname{Span}\left\{m_{\mu}\right\}_{\mu \in \mathcal{P}^{+}, \mu \preceq \lambda}$.

Definition. A linear operator $D: \mathcal{A}^{W} \rightarrow \mathcal{A}^{W}$ is called triangular if $D\left(\mathcal{A}_{\lambda}^{W}\right) \subseteq \mathcal{A}_{\lambda}^{W}$ for all $\lambda \in \mathcal{P}^{+}$.

\section{Determinantal diagonalization}

The triangularity of a linear operator $D$ in $\mathcal{A}^{W}$ reduces its eigenvalue problem to a finite-dimensional one. In this section we diagonalize the triangular operators through a determinantal representation of the eigenfunctions.

Let $D$ be a triangular operator and let $\left\{s_{\lambda}\right\}_{\lambda \in \mathcal{P}^{+}}$be a second basis of $\mathcal{A}^{W}$ that is related to the monomial basis by a unitriangular transformation:

$$
m_{\lambda}=\sum_{\mu \in \mathcal{P}^{+}, \mu \preceq \lambda} a_{\lambda \mu} s_{\mu}, \quad a_{\lambda \lambda}=1
$$




\section{Determinantal CONSTRUCTION OF POLYNOMials}

$\left(\lambda \in \mathcal{P}^{+}\right)$. The triangularity implies that the expansion of $D m_{\lambda}$ in the basis $\left\{s_{\lambda}\right\}_{\lambda \in \mathcal{P}^{+}}$is of the form

$$
D m_{\lambda}=\sum_{\mu \in \mathcal{P}^{+}, \mu \preceq \lambda} b_{\lambda \mu} s_{\mu}, \quad b_{\lambda \lambda}=\epsilon_{\lambda},
$$

with the diagonal matrix elements $\epsilon_{\lambda}, \lambda \in \mathcal{P}^{+}$being precisely the eigenvalues of $D$.

Definition. The triangular operator $D$ is called regular if $\epsilon_{\mu} \neq \epsilon_{\lambda}$ when $\mu \prec \lambda$.

For a regular triangular operator the eigenvalues $\epsilon_{\lambda}, \lambda \in \mathcal{P}^{+}$are semisimple. Let $\left\{p_{\lambda}\right\}_{\lambda \in \mathcal{P}^{+}}$be a corresponding basis of eigenfunctions diagonalizing $D$. Clearly, $p_{\lambda}$ has a monomial expansion of the form

$$
p_{\lambda}=\sum_{\mu \in \mathcal{P}^{+}, \mu \preceq \lambda} c_{\lambda \mu} m_{\mu}, \quad c_{\lambda \lambda}=1,
$$

where we have normalized such that $p_{\lambda}$ is monic. The following theorem provides an explicit determinantal formula for $p_{\lambda}$, given the action of $D$ on $m_{\lambda}$ expressed in the basis $s_{\lambda}$, i.e., given the expansion coefficients $a_{\lambda \mu}$ and $b_{\lambda \mu}$ in Equations (3.1) and (3.2).

Theorem 3.1 (Determinantal formula). Let $D$ be a regular triangular operator in $\mathcal{A}^{W}$ whose action on the monomial symmetric functions is given by Equations (3.1) and (3.2). Then the monic basis $\left\{p_{\lambda}\right\}_{\lambda \in \mathcal{P}^{+}}$of $\mathcal{A}^{W}$ diagonalizing $D$, in the sense that

$$
D p_{\lambda}=\epsilon_{\lambda} p_{\lambda}, \quad \forall \lambda \in \mathcal{P}^{+},
$$

is given explicitly by the (lower) Hessenberg determinant

$$
p_{\lambda}=\frac{1}{\mathcal{E}_{\lambda}}\left|\begin{array}{cccccc}
m_{\lambda^{(1)}} & \epsilon_{\lambda^{(1)}}-\epsilon_{\lambda^{(n)}} & 0 & \ldots & \ldots & 0 \\
m_{\lambda^{(2)}} & d_{\lambda^{(2)} \lambda^{(1)}} & \epsilon_{\lambda^{(2)}}-\epsilon_{\lambda^{(n)}} & 0 & \ldots & 0 \\
\vdots & \vdots & & \ddots & \ddots & \vdots \\
\vdots & \vdots & \vdots & & \ddots & 0 \\
m_{\lambda^{(n-1)}} & d_{\lambda^{(n-1)} \lambda^{(1)}} & d_{\lambda^{(n-1)} \lambda^{(2)}} & \ldots & & \epsilon_{\lambda^{(n-1)}}-\epsilon_{\lambda^{(n)}} \\
m_{\lambda^{(n)}} & d_{\lambda^{(n)} \lambda^{(1)}} & d_{\lambda^{(n)} \lambda^{(2)}} & \ldots & \ldots & d_{\lambda^{(n)} \lambda^{(n-1)}}
\end{array}\right| .
$$

Here $\lambda^{(1)}<\lambda^{(2)}<\cdots<\lambda^{(n-1)}<\lambda^{(n)}=\lambda$ denotes any linear ordering of the dominant weights $\left\{\mu \in \mathcal{P}^{+} \mid \mu \preceq \lambda\right\}$ refining the natural order (2.4), the normalization is determined by

$$
\mathcal{E}_{\lambda}=\prod_{\mu \in \mathcal{P}^{+}, \mu \prec \lambda}\left(\epsilon_{\lambda}-\epsilon_{\mu}\right)
$$

and the matrix elements $d_{\lambda^{(j)} \lambda^{(k)}}(n \geqslant j>k \geqslant 1)$ read

$$
d_{\lambda^{(j)} \lambda^{(k)}}=b_{\lambda^{(j)} \lambda^{(k)}}-\epsilon_{\lambda} a_{\lambda^{(j)} \lambda^{(k)}} .
$$

Proof. Expansion of the determinant with respect to the first column produces a linear combination of monomials in the highest weight space $\mathcal{A}_{\lambda}^{W}$. The coefficient of the leading monomial $m_{\lambda}$ is given by $(-1)^{n-1}$ times the product of the elements on the super-diagonal, which are non-zero by the regularity condition on $D$. Division by $\mathcal{E}_{\lambda}$ thus gives rise to a monic polynomial. It remains to show that this polynomial is an eigenfunction of $D$ with eigenvalue $\epsilon_{\lambda}$. To this end one observes that the action of $\left(D-\epsilon_{\lambda}\right)$ on the determinant affects only its first column. Indeed, we get, upon invoking 


\section{J. F. van Diejen, L. Lapointe And J. Morse}

the expansions (3.1) and (3.2), that

$$
\left(D-\epsilon_{\lambda}\right) p_{\lambda}=\frac{1}{\mathcal{E}_{\lambda}}\left|\begin{array}{ccc}
\ldots & \sum_{k=1}^{j-1} d_{\lambda^{(j)} \lambda^{(k)}} s_{\lambda^{(k)}}+\left(\epsilon_{\lambda^{(j)}}-\epsilon_{\lambda}\right) s_{\lambda^{(j)}} & \ldots \\
\ldots & d_{\lambda^{(j)} \lambda^{(1)}} & \\
\ldots & \vdots & \ldots \\
\ldots & d_{\lambda^{(j)} \lambda^{(j-1)}} & \ldots \\
\ldots & \epsilon_{\lambda^{(j)}}-\epsilon_{\lambda} & \ldots \\
& 0 &
\end{array}\right|
$$

(where, for typographical reasons, we have taken the transpose of our matrix). The latter determinant has a first row of the form $s_{\lambda^{(1)}}$ row $_{2}+s_{\lambda^{(2)}}$ row $_{3}+\cdots+s_{\lambda^{(n-1)}}$ row $_{n}$, and thus vanishes identically.

As a corollary of the determinantal formula for $p_{\lambda}$, one arrives at a linear recurrence relation encoding an efficient algorithm for the computation of the coefficients $c_{\lambda \mu}$ entering the monomial expansion (3.3).

Corollary 3.2 (Linear recurrence relation). The monomial expansion of $p_{\lambda}$ is of the form

$$
p_{\lambda}=\sum_{\ell=1}^{n} c_{\lambda \lambda^{(\ell)}} m_{\lambda^{(\ell)}},
$$

with $c_{\lambda \lambda^{(n)}}=c_{\lambda \lambda}=1$ and

$$
c_{\lambda \lambda^{(\ell-1)}}=\frac{1}{\epsilon_{\lambda}-\epsilon_{\lambda^{(\ell-1)}}} \sum_{k=\ell}^{n} c_{\lambda \lambda^{(k)}} d_{\lambda^{(k)} \lambda^{(\ell-1)}}
$$

$(1<\ell \leqslant n)$.

Proof. Immediate from Theorem 3.1 and the recurrence relation for the evaluation of Hessenberg determinants [Wil88] (see also [LLM00]).

Moreover, by solving the recurrence relation we arrive at the following explicit expression for the coefficients $c_{\lambda \mu}$ of the monomial expansion (3.3).

Corollary 3.3 (Explicit monomial expansion). The coefficients of the monomial expansion $p_{\lambda}=$ $\sum_{\ell=1}^{n} c_{\lambda \lambda^{(\ell)}} m_{\lambda^{(\ell)}}$ are given explicitly by

$$
c_{\lambda \lambda(\ell)} \sum_{\substack{\ell=j_{r}<j_{r-1}<\cdots<j_{1}<j_{0}=n \\ r=1, \ldots, n-\ell}} \frac{d_{\lambda^{\left(j_{0}\right)} \lambda^{\left(j_{1}\right)}} d_{\lambda^{\left(j_{1}\right)} \lambda^{\left(j_{2}\right)}} \cdots d_{\lambda^{\left(j_{r-1}\right)} \lambda^{\left(j_{r}\right)}}}{\left(\epsilon_{\lambda}-\epsilon_{\lambda^{\left(j_{1}\right)}}\right) \cdots\left(\epsilon_{\lambda}-\epsilon_{\lambda^{\left(j_{r}\right)}}\right)},
$$

with the convention that empty sums are equal to $1\left(\right.$ so $\left.c_{\lambda \lambda^{(n)}}=c_{\lambda \lambda}=1\right)$.

\section{Heckman-Opdam polynomials}

In this section we apply the formalism of $\S 3$ to arrive at a determinantal construction of the Heckman-Opdam polynomials for arbitrary (not necessarily reduced) root systems. 


\section{Determinantal CONSTRUCTION OF POLYNOMials}

\subsection{The hypergeometric differential operator}

To a vector $x \in E$ we associate the directional derivation $\partial_{x}$ in $\mathcal{A}$, whose action on the exponential basis is given by

$$
\partial_{x} e^{\lambda}=\langle\lambda, x\rangle e^{\lambda} \quad(\lambda \in \mathcal{P})
$$

Definition [HS94, Opd95]. Let $x_{1}, \ldots, x_{N}$ be an orthonormal basis of $E$ and let $g_{\alpha}, \alpha \in R$ be complex parameters such that $g_{w(\alpha)}=g_{\alpha}$ for all $w \in W$. The second-order partial differential operator

$$
D=\sum_{j=1}^{N} \partial_{x_{j}}^{2}+\sum_{\alpha \in R^{+}} g_{\alpha}\left(\frac{1+e^{-\alpha}}{1-e^{-\alpha}}\right) \partial_{\alpha}
$$

is called the hypergeometric differential operator associated to the root system $R$.

Clearly the definition of $D(4.2)$ does not depend on the particular choice for the orthonormal basis $x_{1}, \ldots, x_{N}$. It is known that the hypergeometric differential operator maps the space of invariants $\mathcal{A}^{W}$ into itself and, furthermore, that it is triangular [HS94, Opd95]. We will now compute the action of $D(4.2)$ on the basis of monomial symmetric functions. To this end some notation is needed. We denote the stabilizer subgroup of $\lambda \in \mathcal{P}$ by $W_{\lambda}=\{w \in W \mid w(\lambda)=\lambda\}$ and the weighted half-sum of the positive roots by $\rho_{g}=\frac{1}{2} \sum_{\alpha \in R^{+}} g_{\alpha} \alpha$. The mapping $r_{\alpha}: E \rightarrow E$ represents the orthogonal reflection in the hyperplane through the origin perpendicular to $\alpha \in R$ (which acts on a vector $x \in E$ as $\left.r_{\alpha}(x)=x-\left\langle x, \alpha^{\vee}\right\rangle \alpha\right)$, and $[p]$ encodes the function that extracts the integral part of a non-negative real number $p$ through truncation.

LEMmA 4.1 (Action of the hypergeometric differential operator). The action of $D(4.2)$ on $m_{\lambda}$, $\lambda \in \mathcal{P}^{+}$, is given by

$$
\begin{aligned}
D m_{\lambda}= & \left(\left\langle\lambda+\rho_{g}, \lambda+\rho_{g}\right\rangle-\left\langle\rho_{g}, \rho_{g}\right\rangle\right) m_{\lambda} \\
& +\frac{1}{\left|W_{\lambda}\right|} \sum_{\alpha \in R^{+}}\left(g_{\alpha}\langle\lambda, \alpha\rangle \sum_{\ell=1}^{\left[\left\langle\lambda, \alpha^{\vee}\right\rangle / 2\right]}\left|W_{\lambda-\ell \alpha}\right|\left|W^{\alpha}(\lambda-\ell \alpha)\right| m_{\lambda-\ell \alpha}\right),
\end{aligned}
$$

where $W^{\alpha} \subset W$ denotes the subgroup of order 2 generated by $r_{\alpha}$ (so $\left|W^{\alpha}(\lambda-\ell \alpha)\right|$ is equal to 1 if $\ell=\left\langle\lambda, \alpha^{\vee}\right\rangle / 2$ and equal to 2 otherwise).

Proof. The computation of the expansion of $D m_{\lambda}$ in the monomial basis hinges on the fundamental identity

$$
\begin{aligned}
\left(\frac{1+e^{-\alpha}}{1-e^{-\alpha}}\right) \partial_{\alpha}\left(e^{\lambda}+e^{r_{\alpha}(\lambda)}\right) & =\langle\lambda, \alpha\rangle e^{\lambda}\left(1+e^{-\alpha}\right)\left(\frac{1-e^{-\left\langle\lambda, \alpha^{\vee}\right\rangle \alpha}}{1-e^{-\alpha}}\right) \\
& =\langle\lambda, \alpha\rangle\left(e^{\lambda}+e^{\lambda-\left\langle\lambda, \alpha^{\vee}\right\rangle \alpha}\right)+2\langle\lambda, \alpha\rangle \sum_{\ell=1}^{\left\langle\lambda, \alpha^{\vee}\right\rangle-1} e^{\lambda-\ell \alpha} \\
& =\langle\lambda, \alpha\rangle\left(e^{\lambda}+e^{r_{\alpha}(\lambda)}\right)+\langle\lambda, \alpha\rangle \sum_{\ell=1}^{\left[\left\langle\lambda, \alpha^{\vee}\right\rangle / 2\right]}\left|W^{\alpha}(\lambda-\ell \alpha)\right|\left(e^{\lambda-\ell \alpha}+e^{r_{\alpha}(\lambda-\ell \alpha)}\right) .
\end{aligned}
$$

Indeed, the following sequence of elementary manipulations reduces the computation of the action 


\section{J. F. van Diejen, L. Lapointe and J. Morse}

of the first-order component of $D(4.2)$ on $m_{\lambda}$ to an application of identity (4.3):

$$
\begin{aligned}
& \sum_{\alpha \in R^{+}} g_{\alpha}\left(\frac{1+e^{-\alpha}}{1-e^{-\alpha}}\right) \partial_{\alpha} m_{\lambda}=\frac{1}{\left|W_{\lambda}\right|} \sum_{\alpha \in R^{+}} g_{\alpha}\left(\frac{1+e^{-\alpha}}{1-e^{-\alpha}}\right) \partial_{\alpha} \sum_{w \in W} e^{w(\lambda)} \\
&=\frac{1}{\left|W_{\lambda}\right|} \sum_{w \in W} w\left(\sum_{\alpha \in R^{+}} g_{\alpha}\left(\frac{1+e^{-\alpha}}{1-e^{-\alpha}}\right) \partial_{\alpha} e^{\lambda}\right) \\
&=\frac{1}{2\left|W_{\lambda}\right|} \sum_{w \in W} w\left(\sum_{\alpha \in R^{+}} g_{\alpha}\left(\frac{1+e^{-\alpha}}{1-e^{-\alpha}}\right) \partial_{\alpha}\left(e^{\lambda}+e^{r_{\alpha}(\lambda)}\right)\right) \\
& \stackrel{\text { Eq. }}{=} \frac{(4.3)}{\mid} \frac{1}{\left|W_{\lambda}\right|} \sum_{w \in W} w\left(g_{\alpha}\langle\lambda, \alpha\rangle e^{\lambda}+g_{\alpha}\langle\lambda, \alpha\rangle \sum_{\alpha \in R^{+}}^{\left[\left\langle\lambda, \alpha^{\vee}\right\rangle / 2\right]}\left|W^{\alpha}(\lambda-\ell \alpha)\right| e^{\lambda-\ell \alpha}\right) \\
&=\sum_{\alpha \in R^{+}} g_{\alpha}\langle\lambda, \alpha\rangle\left(m_{\lambda}+\frac{1}{\left|W_{\lambda}\right|} \sum_{\ell=1}^{\left[\left\langle\lambda, \alpha^{\vee}\right\rangle / 2\right]}\left|W_{\lambda-\ell \alpha}\right|\left|W^{\alpha}(\lambda-\ell \alpha)\right| m_{\lambda-\ell \alpha}\right) .
\end{aligned}
$$

Combined with the action of the second-order component of $D(4.2)$ on $m_{\lambda}$ given by $\sum_{j=1}^{N} \partial_{x_{j}}^{2} m_{\lambda}=$ $\langle\lambda, \lambda\rangle m_{\lambda}$, this produces the formula of the lemma.

It is a standard property of root systems that for any $\lambda \in \mathcal{P}^{+}$the integral convex hull $\mathcal{P}_{\lambda}=\{\mu \in$ $\mathcal{P} \mid W(\mu) \preceq \lambda\}$ is saturated, i.e., if $\mu \in \mathcal{P}_{\lambda}$ then $\mu-\ell \alpha \in \mathcal{P}_{\lambda}$ for every integer $\ell$ between 0 and $\left\langle\mu, \alpha^{\vee}\right\rangle$ (extremal values included) [Hum72]. Hence, it follows from Lemma 4.1 that the hypergeometric differential operator is triangular. To compute for $\mu$ dominant the coefficient of $m_{\mu}$ in $D m_{\lambda}$, it suffices to collect all terms in the lemma for which $\lambda-\ell \alpha \in W(\mu)$. Notice in this connection that for a given $\alpha \in R^{+}$the $\alpha$-string $\lambda-\alpha, \lambda-2 \alpha, \ldots, \lambda-\left[\left\langle\lambda, \alpha^{\vee}\right\rangle / 2\right] \alpha$ may hit the Weyl orbit of $\mu$ at most once. Indeed, it is clear from expanding both sides of the equality $\left\|\lambda-\ell^{\prime} \alpha\right\|^{2}=\|\lambda-\ell \alpha\|^{2}$ that $\lambda-\ell^{\prime} \alpha \in W(\lambda-\ell \alpha)$, with $1 \leqslant \ell^{\prime}, \ell \leqslant\left[\left\langle\lambda, \alpha^{\vee}\right\rangle / 2\right]$, implies $\ell^{\prime}=\ell$.

We thus end up with the following explicit triangular matrix representation of the hypergeometric differential operator with respect to the monomial basis.

Proposition 4.2 (Triangular expansion). Let $\lambda \in \mathcal{P}^{+}$. We have that

$$
D m_{\lambda}=\epsilon_{\lambda} m_{\lambda}+\sum_{\mu \in \mathcal{P}^{+}, \mu \prec \lambda} b_{\lambda \mu} m_{\mu},
$$

with

$$
\begin{aligned}
\epsilon_{\lambda} & =\left\langle\lambda+\rho_{g}, \lambda+\rho_{g}\right\rangle-\left\langle\rho_{g}, \rho_{g}\right\rangle, \\
b_{\lambda \mu} & =\frac{\left|W_{\mu}\right|}{\left|W_{\lambda}\right|} \sum_{\alpha \in[\lambda, \mu]} g_{\alpha}\langle\lambda, \alpha\rangle n_{\lambda \mu}(\alpha) .
\end{aligned}
$$

Here $[\lambda, \mu]$ denotes the subset of roots $\alpha \in R^{+}$for which $\lambda-\ell \alpha \in W(\mu)$ for some (unique) $\ell \in$ $\left\{1,2, \ldots,\left[\left\langle\lambda, \alpha^{\vee}\right\rangle / 2\right]\right\}$, and

$$
n_{\lambda \mu}(\alpha)= \begin{cases}1 & \text { if }\|\mu\|=\left\|P_{\alpha}(\lambda)\right\|, \\ 2 & \text { if }\|\mu\| \neq\left\|P_{\alpha}(\lambda)\right\|,\end{cases}
$$

where $P_{\alpha}=\left(\mathrm{Id}+r_{\alpha}\right) / 2$ is the orthogonal projection onto the hyperplane perpendicular to $\alpha$ through the origin. (So for $\lambda-\ell \alpha \in W(\mu)$ we have that $n_{\lambda \mu}(\alpha)=1$ if $\ell=\left\langle\lambda, \alpha^{\vee}\right\rangle / 2$ and $n_{\lambda \mu}(\alpha)=2$ otherwise.)

For non-negative parameter values the regularity of the hypergeometric differential operator is immediate from the following proposition. 


\section{Determinantal CONSTRUCTION OF POLYNOMials}

Proposition 4.3 (Monotonicity). For non-negative parameters $g_{\alpha}$, the eigenvalues $\epsilon_{\lambda}=\left\langle\lambda+\rho_{g}\right.$, $\left.\lambda+\rho_{g}\right\rangle-\left\langle\rho_{g}, \rho_{g}\right\rangle$ are strictly monotonous in $\lambda \in \mathcal{P}^{+}$, i.e.

$$
\forall \lambda, \mu \in \mathcal{P}^{+}: \quad \mu \prec \lambda \Longrightarrow \epsilon_{\mu}<\epsilon_{\lambda} .
$$

Proof. Assume $\lambda, \mu$ dominant with $\mu \prec \lambda$, and let $\nu=\lambda-\mu$ (so $\left.\nu \in \mathcal{Q}^{+}\right)$. Then

$$
\epsilon_{\lambda}-\epsilon_{\mu}=\langle\nu, \nu\rangle+2\left\langle\mu+\rho_{g}, \nu\right\rangle
$$

which is positive in view of the fact that $\langle\nu, \nu\rangle>0$ and $\left\langle\mu+\rho_{g}, \nu\right\rangle \geqslant 0$ (since both $\mu$ and $\rho_{g}$ lie in the closure of the dominant Weyl chamber).

\subsection{Determinantal construction}

It is known that the eigenbasis diagonalizing the hypergeometric differential operator $D(4.2)$ in $\mathcal{A}^{W}$ is given by the Heckman-Opdam polynomials [HS94, Opd95]. Moreover, since $D$ is regular (Proposition 4.3), and its triangular action on the monomial basis is known explicitly (Proposition 4.2), we can in fact construct this eigenbasis in closed form by means of the determinantal construction in $\S 3$ (with $s_{\lambda}=m_{\lambda}$, so $a_{\lambda \mu}=1$ if $\mu=\lambda$ and $a_{\lambda \mu}=0$ otherwise). This gives rise to the following explicit representation of the Heckman-Opdam polynomials.

Theorem 4.4 (Determinantal construction). For $\lambda \in \mathcal{P}^{+}$, let

$$
p_{\lambda}=m_{\lambda}+\sum_{\mu \in \mathcal{P}^{+}, \mu \prec \lambda} c_{\lambda \mu} m_{\mu}
$$

denote the (monic) Heckman-Opdam polynomial with parameters $g_{\alpha} \geqslant 0$. Then, upon setting for $\mu, \nu \in \mathcal{P}^{+}$

$$
\begin{aligned}
\epsilon_{\mu} & =\left\langle\mu+\rho_{g}, \mu+\rho_{g}\right\rangle-\left\langle\rho_{g}, \rho_{g}\right\rangle, \\
d_{\mu \nu} & =\frac{\left|W_{\nu}\right|}{\left|W_{\mu}\right|} \sum_{\alpha \in[\mu, \nu]} g_{\alpha}\langle\mu, \alpha\rangle n_{\mu \nu}(\alpha),
\end{aligned}
$$

with $[\mu, \nu] \subset R^{+}$and $n_{\mu \nu}(\alpha)$ in accordance with the definition in Proposition 4.2, we have that:

i) the polynomial $p_{\lambda}$ is represented explicitly by the determinantal formula in Theorem 3.1;

ii) the coefficients $c_{\lambda \mu}$ of its monomial expansion are generated by the linear recurrence in Corollary 3.2 ;

iii) the expansion coefficients $c_{\lambda \mu}$ are given in closed form by the formula in Corollary 3.3.

Given a concrete root system $R$, Theorem 4.4 turns into an efficient algorithm for the computation of the associated Heckman-Opdam polynomials. We will illustrate this below for the classical root systems.

Remark (i). The orders of the stabilizers in Theorem 4.4 can be computed by means of Macdonald's formula (see e.g. [Mac01, § 12])

$$
\left|W_{\lambda}\right|=\prod_{\substack{\alpha \in R^{+} \\\left\langle\lambda, \alpha^{\vee}\right\rangle=0}} \frac{\left\langle\rho, \alpha^{\vee}\right\rangle+1+\frac{1}{2} \delta_{\frac{\alpha}{2}}}{\left\langle\rho, \alpha^{\vee}\right\rangle+\frac{1}{2} \delta_{\frac{\alpha}{2}}},
$$

where $\rho=\frac{1}{2} \sum_{\alpha \in R^{+}} \alpha$, and $\delta_{\alpha}=1$ if $\alpha \in R$ and zero otherwise.

Remark (ii). It is clear from the proof of Proposition 4.3 that the hypergeometric differential operator $D$ (4.2) is in fact regular for generic (complex) parameters $g_{\alpha}$ such that $\langle\nu, \nu\rangle+2\left\langle\mu+\rho_{g}, \nu\right\rangle \neq 0$ for all 


\section{J. F. van Diejen, L. Lapointe and J. Morse}

$\nu \in \mathcal{Q}^{+}$and $\mu \in \mathcal{P}^{+}$. Hence, the determinantal construction of the Heckman-Opdam polynomials in Theorem 4.4, as the eigenbasis for the hypergeometric differential operator, extends meromorphically to $g_{\alpha}$ in the complex plane.

Remark (iii). It is known that the coefficients of the Heckman-Opdam polynomials can in principle be computed by means of (cumbersome) Freudenthal type recurrence relations [HS94]. From this perspective, the determinantal construction of Theorem 4.4 thus provides the explicit solution to this Freudenthal type recurrence. The recurrence in part ii of Theorem 4.4 (which arises as a particular case of the general recurrence scheme in Corollary 3.2 upon choosing for our triangular operator the hypergeometric differential operator) reads concretely

$$
\left(\epsilon_{\lambda}-\epsilon_{\lambda^{(\ell-1)}}\right) c_{\lambda \lambda^{(\ell-1)}}=\sum_{k=\ell}^{n} \frac{\left|W_{\lambda^{(\ell-1)}}\right|}{\left|W_{\lambda^{(k)}}\right|} \sum_{\alpha \in\left[\lambda^{(k)}, \lambda^{(\ell-1)}\right]} g_{\alpha}\left\langle\lambda^{(k)}, \alpha\right\rangle n_{\lambda^{(k)} \lambda^{(\ell-1)}}(\alpha) c_{\lambda \lambda^{(k)}} .
$$

It may in fact be seen as a suitable symmetric reduction of the Freudenthal type recurrence relations, enabling their explicit solution in closed form via Corollary 3.3. When $g_{\alpha}=1, \forall \alpha \in R$, our recurrence is closely related to the optimized Freudenthal recurrence scheme for the computation of weight multiplicities of characters of simple Lie groups due to Moody and Patera [MP82].

\subsection{Tables for the classical root systems}

For a concrete root system $R$, the expressions for the matrix elements in the determinantal formula can be compactified considerably. To illustrate this state of affairs, we will now provide tables of these matrix elements for the Heckman-Opdam polynomials associated with the classical root systems. In each case, we will only list the minimum amount of information needed for constructing the matrix, viz., (i) the cone of the dominant weights and its partial order, (ii) the eigenvalues building the super-diagonal of the matrix, and (iii) the values of the lower triangular matrix elements. For further data on the root systems of interest we refer to the tables in Bourbaki [Bou68].

It will be convenient to parameterize the dominant weights of the classical root systems in terms of $N$-tuples

$$
\lambda=\left(\lambda_{1}, \lambda_{2}, \ldots, \lambda_{N}\right)
$$

of weakly decreasing (half-)integers (so $\lambda_{1} \geqslant \lambda_{2} \geqslant \cdots \geqslant \lambda_{N}$ ). Often we think of these $N$-tuples also as multi-sets of the form

$$
\lambda=\left\{\lambda_{1}, \lambda_{2}, \ldots, \lambda_{N}\right\},
$$

where the parts $\lambda_{j}$ are listed from largest to smallest. For $\lambda=\left(\lambda_{1}, \ldots, \lambda_{N}\right)\left(=\left\{\lambda_{1}, \ldots, \lambda_{N}\right\}\right)$, we define $\lambda^{\varepsilon}=\left(\lambda_{1}, \ldots, \lambda_{N-1}, \varepsilon\left|\lambda_{N}\right|\right)\left(=\left\{\lambda_{1}, \ldots, \lambda_{N-1}, \varepsilon\left|\lambda_{N}\right|\right\}\right)$, with $\varepsilon \in\{1,-1\}$. We need the following two operations on our weakly decreasing $N$-tuples:

$$
\begin{aligned}
\lambda \backslash \mu & =\left\{\lambda_{1}, \lambda_{2}, \ldots, \lambda_{N}\right\} \backslash\left\{\mu_{1}, \mu_{2}, \ldots, \mu_{N}\right\}, \\
\lambda \ominus \mu & =\left(\lambda^{+} \backslash \mu^{+},\left(\mu^{+} \backslash \lambda^{+}\right)^{\varepsilon}\right),
\end{aligned}
$$

where $\varepsilon=\operatorname{sign}\left(\lambda_{N}\right) \times \operatorname{sign}\left(\mu_{N}\right)$. The first operation takes the difference of $\lambda$ and $\mu$ as multi-sets, i.e., taking into account the multiplicities of the parts. (By convention, we will list the parts of this difference again from large to small.) The second operation encodes, up to a possible sign, the symmetric difference of $\lambda^{+}$and $\mu^{+}$. For example: $\left(5,3,2 \frac{1}{2}, 1,1\right) \ominus(4,3,3,1,-1)=\left(\left\{5,2 \frac{1}{2}\right\},\{4,-3\}\right)$. Finally, for future reference we furthermore introduce the operations

$$
\begin{aligned}
|\lambda| & =\lambda_{1}+\lambda_{2}+\cdots+\lambda_{N}, \\
\eta_{\lambda}(m) & =\left|\left\{j=1, \ldots, N \mid \lambda_{j}=m \vee \lambda_{j}=-m\right\}\right|, \\
\bar{\lambda} & =\left(\lambda_{1}, \lambda_{2}, \ldots, \lambda_{N-1},-\lambda_{N}\right),
\end{aligned}
$$




\section{Determinantal CONSTRUCTION OF POLYNOMials}

producing, respectively, the sum of the parts, the number of parts with specified absolute value $|m|$, and the conjugate $N$-tuple with the sign of the last part flipped.

4.3.1 The case $R=A_{N-1}$. For the type $A$ root system the Heckman-Opdam polynomials reduce (in essence) to Jack polynomials [Sta89, Mac95]. The determinantal construction in Theorem 4.4 reproduces in this particular case the determinantal construction of the Jack polynomials found by Lapointe, Lascoux and Morse [LLM00].

In dealing with the type $A$ root system, it is more convenient to work with partitions rather than with the weights themselves. Let $\Lambda_{N}$ be the set of partitions with at most $N$ parts, i.e., the set of weakly decreasing $N$-tuples with components given by non-negative integers. For $\lambda, \mu \in \Lambda_{N}$ the dominance order on these partitions is defined as

$$
\lambda \succeq \mu \Longleftrightarrow|\lambda|=|\mu| \text { and } \sum_{j=1}^{k}\left(\lambda_{j}-\mu_{j}\right) \geqslant 0 \quad \text { for } k=1, \ldots, N-1 .
$$

We write $\hat{\lambda}$ for the orthogonal projection of $\lambda \in \Lambda_{N}$ onto the hyperplane $E \subset \mathbb{R}^{N}$ perpendicular to the vector $(1,1, \ldots, 1)$ :

$$
\hat{\lambda}=\left(\lambda_{1}, \ldots, \lambda_{N}\right)-\frac{|\lambda|}{N}(1, \ldots, 1) .
$$

The cone of dominant weights associated to the root system $A_{N-1}$ is now given by the projection of $\Lambda_{N}$ onto the hyperplane $E$, i.e. $\mathcal{P}_{A}^{+}=\left\{\hat{\lambda} \mid \lambda \in \Lambda_{N}\right\}$, equipped with a partial order induced by the dominance ordering of the partitions in Equation (4.8). Specifically, for given $\lambda \in \Lambda_{N}$ all dominant weights smaller than or equal to $\hat{\lambda} \in \mathcal{P}_{A}^{+}$are given by

$$
\mathcal{P}_{\hat{\lambda}, A}^{+}=\left\{\hat{\mu} \mid \mu \in \Lambda_{N} \wedge \mu \preceq \lambda\right\} .
$$

The projection $\lambda \rightarrow \hat{\lambda}$ (4.9) has a non-trivial kernel of the form $(1,1, \ldots, 1) \mathbb{N}$. The set in Equation (4.10), however, is clearly independent of the particular choice for the partition $\lambda$ projecting onto the dominant weight $\hat{\lambda}$.

The Weyl group acts transitively on the root system $A_{N-1}$, as all roots have the same length. Thus, the value of the root multiplicity parameter $g_{\alpha}$ does not depend on $\alpha$, viz. $g_{\alpha}=g$ for all $\alpha \in R$. Given a partition $\lambda \in \Lambda_{N}$, let us define for $\mu \preceq \lambda$

$$
\epsilon_{\mu}^{A}=\sum_{j=1}^{N} \mu_{j}\left(\mu_{j}+g(N+1-2 j)\right)
$$

and for $\nu \prec \mu \preceq \lambda$

$$
d_{\mu \nu}^{A}= \begin{cases}2 g\left(m_{1}-m_{2}\right) \mathcal{N}_{\nu}\left(n_{1}, n_{2}\right) & \text { if } \mu \ominus \nu=\left(\left\{m_{1}, m_{2}\right\},\left\{n_{1}, n_{2}\right\}\right) \text { with } m_{1}-n_{1}=n_{2}-m_{2}>0 \\ 0 & \text { otherwise }\end{cases}
$$

where

$$
\mathcal{N}_{\nu}\left(n_{1}, n_{2}\right)= \begin{cases}\eta_{\nu}\left(n_{1}\right) \eta_{\nu}\left(n_{2}\right) & \text { if }\left|n_{1}\right| \neq\left|n_{2}\right| \\
\left(\begin{array}{c}
\eta_{\nu}\left(n_{1}\right) \\
2
\end{array}\right) & \text { if }\left|n_{1}\right|=\left|n_{2}\right|\end{cases}
$$

and $\eta_{\nu}(n)$ denotes the multiplicity counter defined in Equation $(4.7 \mathrm{~b})$. The super-diagonal $\epsilon_{\hat{\mu}}^{A}-\epsilon_{\hat{\lambda}}^{A}$ $(\hat{\mu} \preceq \hat{\lambda})$ and the lower triangular block $d_{\hat{\mu} \hat{\nu}}^{A}(\hat{\nu} \prec \hat{\mu} \preceq \hat{\lambda})$ of the Hessenberg matrix in Theorem 4.4 become for the $A_{N-1}$-type Heckman-Opdam polynomial $p_{\hat{\lambda}}^{A}$ :

$$
\epsilon_{\hat{\mu}}^{A}-\epsilon_{\hat{\lambda}}^{A}=\epsilon_{\mu}^{A}-\epsilon_{\lambda}^{A} \quad \text { and } \quad d_{\hat{\mu} \hat{\nu}}^{A}=d_{\mu \nu}^{A},
$$




\section{J. F. VAn Diejen, L. Lapointe and J. Morse}

respectively. (Notice in this connection that the expressions $\epsilon_{\mu}^{A}-\epsilon_{\lambda}^{A}$ and $d_{\mu \nu}^{A}$ on the right-hand side are invariant with respect to the additive action of $(1,1, \ldots, 1) \mathbb{N}$ on $\Lambda_{N}$.)

4.3.2 The case $D_{N}$. The cone of dominant weights $\mathcal{P}_{D}^{+}$consists of the $N$-tuples $\lambda=\left(\lambda_{1}, \ldots, \lambda_{N}\right)$ with parts $\lambda_{j}$ that are all integers or all half-integers subject to the ordering

$$
\lambda_{1} \geqslant \lambda_{2} \geqslant \cdots \geqslant \lambda_{N-1} \geqslant\left|\lambda_{N}\right| \text {. }
$$

The partial order on $\mathcal{P}_{+}^{D}$ is defined as

$$
\lambda \succeq \mu \Longleftrightarrow \begin{cases}\sum_{j=1}^{k}\left(\lambda_{j}-\mu_{j}\right) \in \mathbb{N} & \text { for } k=1, \ldots, N-2, \\ \sum_{j=1}^{N-1}\left(\lambda_{j}-\mu_{j}\right)+\varepsilon\left(\lambda_{N}-\mu_{N}\right) \in 2 \mathbb{N} & \text { for } \varepsilon= \pm 1 .\end{cases}
$$

The Weyl group again acts transitively on the root system $D_{N}$, so we have $g_{\alpha}=g, \forall \alpha \in R$. The super-diagonal $\epsilon_{\mu}^{D}-\epsilon_{\lambda}^{D}(\mu \preceq \lambda)$ and the lower triangular block $d_{\mu \nu}^{D}(\nu \prec \mu \preceq \lambda)$ of the Hessenberg matrix in Theorem 4.4 become for the $D_{N}$-type Heckman-Opdam polynomial $p_{\lambda}^{D}$ of the form

$$
\epsilon_{\mu}^{D}=\sum_{j=1}^{N} \mu_{j}\left(\mu_{j}+2 g(N-j)\right)
$$

and

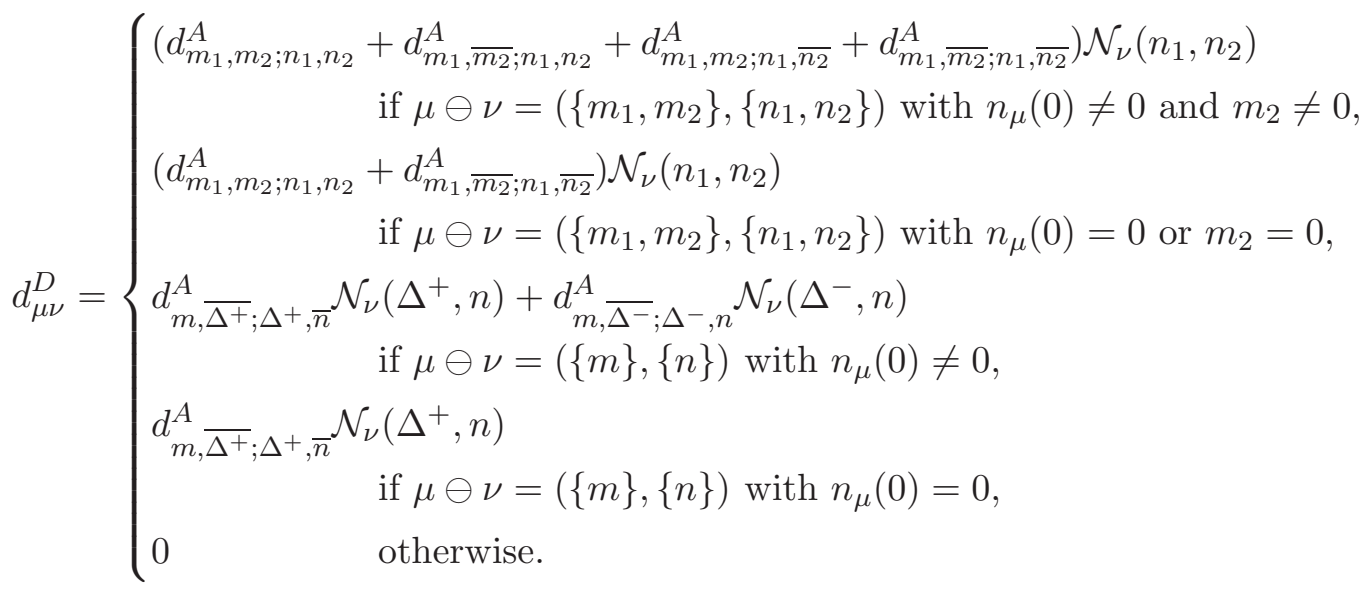

Here $d_{m_{1}, m_{2} ; n_{1}, n_{2}}^{A}$ refers to the $A_{1}$-type matrix elements (cf. Equation (4.11b)), namely,

$$
d_{m_{1}, m_{2} ; n_{1}, n_{2}}^{A}= \begin{cases}2 g\left(m_{1}-m_{2}\right) & \text { if } m_{1}-n_{1}=n_{2}-m_{2}>0 \\ 0 & \text { otherwise }\end{cases}
$$

and $\mathcal{N}_{\nu}\left(n_{1}, n_{2}\right)$ is the same as above (cf. Equation (4.12)). Furthermore, $\bar{m}$ stands for $-m$ and $\Delta^{ \pm}=(m \pm n) / 2$.

Remark. In the first line of $d_{\mu \nu}^{D}$, at most two terms can be non-zero if $n_{2}=0$, and at most one term otherwise. Similarly, in the second line, at most one term can be non-zero.

4.3.3 The case $B_{N}$. The cone of dominant weights $\mathcal{P}_{B}^{+}$consists of the $N$-tuples $\lambda=\left(\lambda_{1}, \ldots, \lambda_{N}\right)$ with parts $\lambda_{j}$ that are all integers or all half-integers subject to the ordering

$$
\lambda_{1} \geqslant \lambda_{2} \geqslant \cdots \geqslant \lambda_{N-1} \geqslant \lambda_{N} \geqslant 0 \text {. }
$$




\section{Determinantal CONSTRUCTION OF POLYNOMials}

The partial order on $\mathcal{P}_{B}^{+}$is defined as

$$
\lambda \succeq \mu \Longleftrightarrow \sum_{j=1}^{k}\left(\lambda_{j}-\mu_{j}\right) \in \mathbb{N} \quad \text { for } k=1, \ldots, N .
$$

The $B_{N}$-type root system has two root lengths, so the action of the Weyl group splits up in two orbits. We will denote the root multiplicity parameters for the long and short roots by $g$ and $g_{s}$, respectively. The super-diagonal $\epsilon_{\mu}^{B}-\epsilon_{\lambda}^{B}(\mu \preceq \lambda)$ and the lower triangular block $d_{\mu \nu}^{B}(\nu \prec \mu \preceq \lambda)$ of the Hessenberg matrix in Theorem 4.4 become for the $B_{N}$-type Heckman-Opdam polynomial $p_{\lambda}^{B}$ of the form

$$
\epsilon_{\mu}^{B}=\sum_{j=1}^{N} \mu_{j}\left(\mu_{j}+2 g(N-j)+g_{s}\right)
$$

and

$$
d_{\mu \nu}^{B}= \begin{cases}d_{\mu \nu}^{D}+d_{\mu \nu}^{\text {short }} & \text { if } \mu=\bar{\mu} \\ d_{\mu \nu}^{D}+d_{\bar{\mu} \nu}^{D}+d_{\mu \nu}^{\text {short }} & \text { if } \mu \neq \bar{\mu}\end{cases}
$$

with $d_{\mu \nu}^{D}$ taken from Equation (4.16b) and

$$
d_{\mu \nu}^{\text {short }}= \begin{cases}2 g_{s} m \eta_{\nu}(n) & \text { if } \mu \ominus \nu=(\{m\},\{n\}) \text { with } m-n>0 \\ 0 & \text { otherwise. }\end{cases}
$$

4.3.4 The cases $C_{N}$ and $B C_{N}$. The cone of dominant weights $\mathcal{P}_{B C}^{+}$consists of the partitions $\lambda=\left(\lambda_{1}, \ldots, \lambda_{N}\right)$ in $\Lambda_{N}$ (cf. the $A_{N-1}$-type above). The partial order on $\mathcal{P}_{B C}^{+}$is the same as in the $B_{N}$-case (cf. Equation (4.19))

$$
\lambda \succeq \mu \Longleftrightarrow \sum_{j=1}^{k}\left(\lambda_{j}-\mu_{j}\right) \geqslant 0 \quad \text { for } k=1, \ldots, N .
$$

The $B C_{N}$-type root system has three root lengths, so the action of the Weyl group splits up into three orbits. We will denote the root multiplicity parameters for the long and short roots by $g_{l}$ and $g_{s}$, respectively. The parameter for the remaining (i.e. middle) roots is $g$. The super-diagonal $\epsilon_{\mu}^{B C}-\epsilon_{\lambda}^{B C}(\mu \preceq \lambda)$ and the lower triangular block $d_{\mu \nu}^{B C}(\nu \prec \mu \preceq \lambda)$ of the Hessenberg matrix in Theorem 4.4 become for the $B C_{N}$-type Heckman-Opdam polynomial $p_{\lambda}^{B C}$ of the form

$$
\epsilon_{\mu}^{B C}=\sum_{j=1}^{N} \mu_{j}\left(\mu_{j}+2 g(N-j)+g_{s}+2 g_{l}\right)
$$

and

$$
d_{\mu \nu}^{B C}= \begin{cases}d_{\mu \nu}^{D}+d_{\mu \nu}^{\text {short }}+d_{\mu \nu}^{\text {long }} & \text { if } \mu=\bar{\mu}, \\ d_{\mu \nu}^{D}+d_{\bar{\mu} \nu}^{D}+d_{\mu \nu}^{\text {short }}+d_{\mu \nu}^{\text {long }} & \text { if } \mu \neq \bar{\mu},\end{cases}
$$

where $d_{\mu \nu}^{D}$ and $d_{\mu \nu}^{\text {short }}$ are taken from Equations (4.16b) and (4.21), respectively, and

$$
d_{\mu \nu}^{\text {long }}= \begin{cases}4 g_{l} m \eta_{\nu}(n) & \text { if } \mu \ominus \nu=(\{m\},\{n\}) \text { with } m-n \in 2 \mathbb{N}, \\ 0 & \text { otherwise. }\end{cases}
$$

Remark (i). The $C_{N}$ case is obtained from the $B C_{N}$ case by setting $g_{s}=0$. In this situation one generally can reduce the size of the Hessenberg matrix, as the partial order on the weights for the 


\section{J. F. van Diejen, L. Lapointe and J. Morse}

$C_{N}$ root system, viz.

$$
\lambda \succeq \mu \Longleftrightarrow\left\{\begin{array}{l}
\sum_{j=1}^{k}\left(\lambda_{j}-\mu_{j}\right) \in \mathbb{N} \quad \text { for } k=1, \ldots, N-1, \\
\sum_{j=1}^{N}\left(\lambda_{j}-\mu_{j}\right) \in 2 \mathbb{N},
\end{array}\right.
$$

is less refined than the partial order in Equation (4.19) corresponding to the $B C_{N}$ root system. More specifically, if the monomial on the $l$ th row is not comparable to the leading monomial in the $C_{N}$ ordering (4.25), then we may eliminate (for $g_{s}=0$ ) the $l$ th row together with the $(l+1)$ th column from the Hessenberg matrix. (To keep the normalization monic, we should of course also delete the corresponding factors from the normalization constant $\mathcal{E}_{\lambda}$.)

Example. For $R=B_{3}$ and $\lambda=(2,1,0)$ the determinantal formula reads

$$
\begin{aligned}
p_{2,1,0}= & \left((2+4 g)\left(1+2 g+g_{s}\right)\left(3+4 g+g_{s}\right)\left(4+6 g+2 g_{s}\right)\left(5+10 g+3 g_{s}\right)\right)^{-1} \\
& \times\left|\begin{array}{llcccc}
m_{0,0,0} & -5-10 g-3 g_{s} & 0 & 0 & 0 & 0 \\
m_{1,0,0} & 6 g_{s} & -4-6 g-2 g_{s} & 0 & 0 & 0 \\
m_{1,1,0} & 24 g & 4 g_{s} & -3-4 g-g_{s} & 0 & 0 \\
m_{2,0,0} & 12 g_{s} & 4 g_{s} & 4 g & -1-2 g-g_{s} & 0 \\
m_{1,1,1} & 0 & 8 g & 2 g_{s} & 0 & -2-4 g \\
m_{2,1,0} & 0 & 24 g+8 g_{s} & 8 g_{s} & 4 g_{s} & 12 g
\end{array}\right| .
\end{aligned}
$$

This polynomial may also be interpreted as a special case of the $B C_{3}$-type Heckman-Opdam polynomial $p_{2,1,0}$ with $g_{l}=0$. We observe in this connection that for $g_{s}=0$, the 1 st, the $3 \mathrm{rd}$ and the 4th row, together with the 2nd, the 4th and the 5th column, may be eliminated from the Hessenberg matrix (cf. Remark (i) above). Indeed, the weights $(0,0,0),(1,1,0)$ and $(2,0,0)$ are not comparable to the highest weight $(2,1,0)$ with respect to the $C_{N}$-type partial order in Equation (4.25). (To keep our normalization monic, we must also delete the 2 nd, the 3rd and the 5th factor from the normalization constant.)

\section{Macdonald Polynomials: the case $t_{\alpha}=t$}

In this section we apply the formalism of $\S 3$ to arrive at a determinantal construction of the Macdonald polynomials. Throughout this section it will be assumed that the root system $R$ is reduced and that the dual root system $R^{\vee}$ has a minuscule weight (thus including the types $A_{N}, B_{N}$, $C_{N}, D_{N}, E_{6}$ and $E_{7}$ while excluding the types $B C_{n}, E_{8}, F_{4}$ and $G_{2}$ ). The Macdonald polynomials depend on complex parameters $q$ and $t_{\alpha}, \alpha \in R$ such that $t_{w(\alpha)}=t_{\alpha}$ for all $w \in W$. In this section we will restrict to the case that $t_{\alpha}=t, \forall \alpha \in R$ and, unless explicitly stated otherwise, we will consider the $(q, t)$ parameters as indeterminates rather than real (or complex) numbers.

\subsection{The Macdonald operator}

For $x \in E$, we define the $q$-translation in $\mathcal{A}$ via its action on the exponential basis:

$$
T_{x, q} e^{\lambda}=q^{\langle\lambda, x\rangle} e^{\lambda} \quad(\lambda \in \mathcal{P}) .
$$

Definition [Mac98, Mac01]. Let $\pi$ be a minuscule weight for $R^{\vee}$, i.e., the vector $\pi \in E$ is such that $\langle\pi, \alpha\rangle \in\{0,1\}$ for all $\alpha \in R^{+}$. The $q$-difference operator

$$
D_{\pi}=\frac{1}{\left|W_{\pi}\right|} \sum_{w \in W}\left(\prod_{\alpha \in R^{+}} \frac{1-t^{\langle\pi, \alpha\rangle} e^{w(\alpha)}}{1-e^{w(\alpha)}}\right) T_{w(\pi), q}
$$

is called the Macdonald operator associated to the minuscule weight $\pi$. 


\section{Determinantal CONSTRUCTION OF POLYNOMials}

(The above definition of the Macdonald operator $D_{\pi}$ is not precisely the same as the one employed by Macdonald [Mac98, Mac01]; both definitions do coincide upon restriction to the space of invariant polynomials $\mathcal{A}^{W}$ though.)

In order to compute the action of $D_{\pi}$ on the monomial basis we will make use of the Weyl characters $\chi_{\lambda}, \lambda \in \mathcal{P}$ :

$$
\chi_{\lambda}=\delta^{-1} \sum_{w \in W} \operatorname{det}(w) e^{w(\lambda+\rho)},
$$

where $\rho$ and $\delta$ denote the half sum of the positive roots and the Weyl denominator respectively

$$
\begin{aligned}
& \rho=\frac{1}{2} \sum_{\alpha \in R^{+}} \alpha, \\
& \delta=\prod_{\alpha \in R^{+}}\left(e^{\alpha / 2}-e^{-\alpha / 2}\right)=\sum_{w \in W} \operatorname{det}(w) e^{w(\rho)} .
\end{aligned}
$$

(Clearly the determinant $\operatorname{det}(w)$ is equal to $(-1)^{\ell(w)}$, where $\ell(w)$ represents the length of the (shortest) decomposition of $w$ into a product of simple reflections.) It is well known that for $\lambda \in \mathcal{P}^{+}$one has that

$$
\chi_{\lambda}=\sum_{\mu \in \mathcal{P}^{+}, \mu \preceq \lambda} K_{\lambda \mu} m_{\mu}, \quad K_{\lambda \lambda}=1,
$$

with coefficients $K_{\lambda \mu} \in \mathbb{N}$. (In fact, the coefficients $K_{\lambda \mu}$, which are also known as Kostka numbers, count the multiplicity of the weight $\mu$ in the irreducible representation of the Lie algebra corresponding to the root system $R$ with highest weight $\lambda$.) An efficient way to compute the coefficients $K_{\lambda \mu}$ is through the application of Theorem 4.4 with $g_{\alpha}=1, \forall \alpha \in R$. However, for our purposes such a calculation is not necessary as we need the inverse of this basis transformation rather than Equation (5.4) itself (cf. Corollary 5.3 below).

It is evident from the expansion in Equation (5.4) that the Weyl characters $\left\{\chi_{\lambda}\right\}_{\lambda \in \mathcal{P}^{+}}$form a basis of $\mathcal{A}^{W}$. The following lemma provides a formula for the action of the Macdonald operator $D_{\pi}$ on the monomials $m_{\lambda}$ in terms of Weyl characters. For the root system $R=A_{N}$ the formula in question is due to Macdonald [Mac95, Mac98].

Lemma 5.1 (Action of the Macdonald operator). Let $\lambda \in \mathcal{P}^{+}$. Then one has that

$$
D_{\pi} m_{\lambda}=t^{\langle\pi, \rho\rangle} \sum_{\nu \in W(\lambda)}\left(\sum_{\tau \in W(\pi)} t^{\langle\tau, \rho\rangle} q^{\langle\tau, \nu\rangle}\right) \chi_{\nu}
$$

Proof. Our starting point is the Weyl denominator formula in the form

$$
e^{-\rho} \prod_{\alpha \in R^{+}}\left(e^{\alpha}-1\right)=\sum_{w \in W} \operatorname{det}(w) e^{w(\rho)} .
$$

By acting on both sides with the $t$-translator $T_{\pi, t}$ we obtain

$$
t^{-\langle\pi, \rho\rangle} e^{-\rho} \prod_{\alpha \in R^{+}}\left(t^{\langle\pi, \alpha\rangle} e^{\alpha}-1\right)=\sum_{w \in W} \operatorname{det}(w) t^{\langle\pi, w(\rho)\rangle} e^{w(\rho)} .
$$

Division of the latter identity by the former gives rise to the following expansion for the coefficients of the Macdonald operator

$$
\prod_{\alpha \in R^{+}} \frac{1-t^{\langle\pi, \alpha\rangle} e^{\alpha}}{1-e^{\alpha}}=\delta^{-1} t^{\langle\pi, \rho\rangle} \sum_{w \in W} \operatorname{det}(w) t^{\langle\pi, w(\rho)\rangle} e^{w(\rho)} .
$$

Substitution of this expansion in the definition of $D_{\pi}$ (taking into account the anti-symmetry of the 


\section{J. F. van Diejen, L. Lapointe and J. Morse}

Weyl denominator $w(\delta)=\operatorname{det}(w) \delta)$, and acting on the exponential $e^{\nu}$ yields

$$
\begin{aligned}
D_{\pi} e^{\nu} & =\delta^{-1} \frac{t^{\langle\pi, \rho\rangle}}{\left|W_{\pi}\right|} \sum_{w_{1}, w_{2} \in W} \operatorname{det}\left(w_{1} w_{2}\right) t^{\left\langle\pi, w_{2}(\rho)\right\rangle} q^{\left\langle w_{1}(\pi), \nu\right\rangle} e^{\nu+w_{1} w_{2}(\rho)} \\
& =\delta^{-1} \frac{t^{\langle\pi, \rho\rangle}}{\left|W_{\pi}\right|} \sum_{w_{1}, w_{2} \in W} \operatorname{det}\left(w_{1} w_{2}\right) t^{\left\langle w_{1}(\pi), w_{1} w_{2}(\rho)\right\rangle} q^{\left\langle w_{1}(\pi), \nu\right\rangle} e^{\nu+w_{1} w_{2}(\rho)} \\
& \stackrel{w_{1} w_{2} \rightarrow w}{=} \delta^{-1} t^{\langle\pi, \rho\rangle} \sum_{w \in W, \tau \in W(\pi)} \operatorname{det}(w) t^{\langle\tau, w(\rho)\rangle} q^{\langle\tau, \nu\rangle} e^{\nu+w(\rho)} .
\end{aligned}
$$

Summation over $\nu$ in the orbit $W(\lambda)$ then entails

$$
\begin{aligned}
D_{\pi} m_{\lambda} & =\delta^{-1} t^{\langle\pi, \rho\rangle} \sum_{\substack{w \in W \\
\tau \in W(\pi), \nu \in W(\lambda)}} \operatorname{det}(w) t^{\langle\tau, w(\rho)\rangle} q^{\langle\tau, \nu\rangle} e^{\nu+w(\rho)} \\
& =\delta^{-1} t^{\langle\pi, \rho\rangle} \sum_{\substack{w \in W \\
\tau \in W(\pi), \nu \in W(\lambda)}} \operatorname{det}(w) t^{\langle\tau, w(\rho)\rangle} q^{\langle\tau, w(\nu)\rangle} e^{w(\nu+\rho)} \\
& =t^{\langle\pi, \rho\rangle} \sum_{\substack{\tau \in W(\pi) \\
\nu \in W(\lambda)}} t^{\langle\tau, \rho\rangle} q^{\langle\tau, \nu\rangle} \chi_{\nu},
\end{aligned}
$$

which completes the proof.

For $\lambda \in \mathcal{P}$, let $w_{\lambda}$ be the unique shortest Weyl group element such that $w_{\lambda}(\lambda) \in \mathcal{P}^{+}$. Then it follows from the definition of the Weyl characters that for $\nu \in \mathcal{P}$

$$
\chi_{\nu}= \begin{cases}\operatorname{det}\left(w_{\nu+\rho}\right) \chi_{\left(w_{\nu+\rho}(\nu+\rho)-\rho\right)} & \text { if }\left|W_{\nu+\rho}\right|=1, \\ 0 & \text { if }\left|W_{\nu+\rho}\right|>1 .\end{cases}
$$

(Notice in this connection that, in view of Equation (4.4), the stabilizer of a weight is non-trivial if and only if there exists a root $\alpha \in R^{+}$perpendicular to it, i.e., if and only if there exists a reflection $r_{\alpha}, \alpha \in R^{+}$stabilizing the weight in question.) Hence, to find for $\lambda, \mu \in \mathcal{P}^{+}$the multiplicity of $\chi_{\mu}$ in $D_{\pi} m_{\lambda}$, we have to collect all terms in the formula of Lemma 5.1 corresponding to weights $\nu \in W(\lambda)$ such that $w_{\nu+\rho}(\nu+\rho)-\rho=\mu$, or equivalently, $\nu=w_{\nu+\rho}^{-1}(\mu+\rho)-\rho$. Clearly the action of $D_{\pi}$ is triangular [Mac98, Mac01], since

$$
\mu=w_{\nu+\rho}(\nu+\rho)-\rho=w_{\nu+\rho}(\nu)-\left(\rho-w_{\nu+\rho}(\rho)\right) \preceq w_{\nu+\rho}(\nu) \preceq w_{\nu}(\nu)=\lambda
$$

(where in the two last steps we used the fact that any dominant weight $\lambda$ is maximal in its Weyl orbit, i.e. $w(\lambda) \preceq \lambda$ for all $w \in W$ [Hum72]). We thus arrive at the following explicit triangular expansion of $D_{\pi} m_{\lambda}$ in terms of $\chi_{\mu}$.

Proposition 5.2 (Triangular expansion). Let $\lambda \in \mathcal{P}^{+}$. We have that

$$
D_{\pi} m_{\lambda}=\epsilon_{\lambda} \chi_{\lambda}+\sum_{\mu \in \mathcal{P}^{+}, \mu \prec \lambda} b_{\lambda \mu} \chi_{\mu},
$$

with

$$
\begin{aligned}
\epsilon_{\lambda} & =t^{\langle\pi, \rho\rangle} \sum_{\tau \in W(\pi)} t^{\langle\tau, \rho\rangle} q^{\langle\tau, \lambda\rangle}, \\
b_{\lambda \mu} & =\sum_{\nu \in W(\lambda) \cap(W(\mu+\rho)-\rho)} \operatorname{det}\left(w_{\rho+\nu}\right) \epsilon_{\nu} .
\end{aligned}
$$




\section{DETERminantal CONSTRUCTION OF POLYNOMIALS}

For $t=1$ the Macdonald operator $D_{\pi}$ trivializes to $\sum_{\tau \in W(\pi)} T_{\tau, q}$, which acts diagonally on $m_{\lambda}$ through multiplication by the eigenvalue $\sum_{\tau \in W(\pi)} q^{\langle\tau, \lambda\rangle}$. The formula of Lemma 5.1 reduces in this case (and upon division by the eigenvalue) to the following well-known relation between the symmetric monomials and the Weyl characters:

$$
m_{\lambda}=\sum_{\mu \in W(\lambda)} \chi_{\mu} .
$$

In the same way, one recovers from Proposition 5.2 the inverse of the expansion in Equation (5.4).

Corollary 5.3 (Inverse Kostka numbers). Let $\lambda \in \mathcal{P}^{+}$. The expansion of the symmetric monomial $m_{\lambda}$ in terms of Weyl characters is given by

$$
m_{\lambda}=\chi_{\lambda}+\sum_{\mu \in \mathcal{P}^{+}, \mu \prec \lambda} a_{\lambda \mu} \chi_{\mu}
$$

with

$$
a_{\lambda \mu}=\sum_{\nu \in W(\lambda) \cap(W(\mu+\rho)-\rho)} \operatorname{det}\left(w_{\rho+\nu}\right) .
$$

The following proposition guarantees that the Macdonald operator $D_{\pi}$ is regular.

Proposition 5.4 (Regularity). The Macdonald operator $D_{\pi}$ is regular in the sense that

$$
\forall \lambda, \mu \in \mathcal{P}^{+}: \quad \mu \prec \lambda \Longrightarrow \epsilon_{\mu}(q, t) \neq \epsilon_{\lambda}(q, t)
$$

(as (analytic) functions of the indeterminates $q$ and $t$ ).

Proof. After setting $t=q^{g}$ and $q=\exp (z)$, we get

$$
\begin{aligned}
t^{-\langle\pi, \rho\rangle} \epsilon_{\lambda} & =\sum_{\tau \in W(\pi)} \exp (z\langle\tau, \lambda+g \rho\rangle) \\
& =|W(\pi)|+z \sum_{\tau \in W(\pi)}\langle\tau, \lambda+g \rho\rangle+\frac{z^{2}}{2} \sum_{\tau \in W(\pi)}\langle\tau, \lambda+g \rho\rangle^{2}+O\left(z^{3}\right) \\
& =|W(\pi)|+c_{\pi} z^{2}\langle\lambda+g \rho, \lambda+g \rho\rangle+O\left(z^{3}\right),
\end{aligned}
$$

with $c_{\pi}>0$. (In the last step we employed the fact that the $W$-invariant linear form $\sum_{\tau \in W(\pi)}\langle\tau, x\rangle$ vanishes and that the $W$-invariant positive quadratic form $\sum_{\tau \in W(\pi)}\langle\tau, x\rangle^{2}$ must be proportional to $\langle x, x\rangle$, because the representation of the Weyl group on $E$ is irreducible and unitary). When $g$ is positive, one has that $\langle\mu+g \rho, \mu+g \rho\rangle\langle\langle\lambda+g \rho, \lambda+g \rho\rangle$ for all dominant weights $\mu, \lambda$ with $\mu \prec \lambda$ in view of Proposition 4.3. It thus follows that for comparable dominant weights $\mu \neq \lambda$ the corresponding eigenvalues $\epsilon_{\mu}(q, t)$ and $\epsilon_{\lambda}(q, t)$ cannot be equal as (analytic) functions of the indeterminates $q$ and $t$.

\subsection{Determinantal construction}

A key result in Macdonald's seminal work [Mac01] states that the Macdonald polynomials form the eigenbasis diagonalizing $D_{\pi}(5.2)$ in $\mathcal{A}^{W}$. We will now apply the determinantal formalism of $\S 3$ to construct this eigenbasis. To this end we pick for the second basis $\left\{s_{\lambda}\right\}_{\lambda \in \mathcal{P}^{+}}$the basis of Weyl characters $\left\{\chi_{\lambda}\right\}_{\lambda \in \mathcal{P}^{+}}$. Specifically, by plugging in the eigenvalues $\epsilon_{\lambda}$ and off-diagonal matrix elements $b_{\lambda \mu}$ from Proposition 5.2, together with the inverse Kostka numbers $a_{\lambda \mu}$ from Corollary 5.3, the formulas of Theorem 3.1 and the Corollaries 3.2 and 3.3 give rise to the desired eigenbasis of the corresponding Macdonald operator $D_{\pi}$. We thus end up with the following determinantal construction of the Macdonald polynomials. 


\section{J. F. van Diejen, L. Lapointe And J. Morse}

Theorem 5.5 (Determinantal construction). For $\lambda \in \mathcal{P}^{+}$, let

$$
p_{\lambda}=m_{\lambda}+\sum_{\mu \in \mathcal{P}^{+}, \mu \prec \lambda} c_{\lambda \mu} m_{\mu}
$$

denote the (monic) Macdonald polynomial with $t_{\alpha}=t, \forall \alpha \in R$. Then, upon setting for $\mu, \nu \in \mathcal{P}^{+}$

$$
\begin{aligned}
\epsilon_{\mu} & =t^{\langle\pi, \rho\rangle} \sum_{\tau \in W(\pi)} t^{\langle\tau, \rho\rangle} q^{\langle\tau, \mu\rangle}, \\
d_{\mu \nu} & =\sum_{\kappa \in W(\mu) \cap(W(\nu+\rho)-\rho)} \operatorname{det}\left(w_{\rho+\kappa}\right)\left(\epsilon_{\kappa}-\epsilon_{\lambda}\right)
\end{aligned}
$$

(so $\left.d_{\mu \mu}=\epsilon_{\mu}-\epsilon_{\lambda}\right)$, we have that:

i) the polynomial $p_{\lambda}$ is represented explicitly by the determinantal formula in Theorem 3.1;

ii) the coefficients $c_{\lambda \mu}$ of its monomial expansion are generated by the linear recurrence in Corollary 3.2;

iii) the expansion coefficients $c_{\lambda \mu}$ are given in closed form by the formula in Corollary 3.3.

Remark (i). It is well known that for $t=q^{g}$ and $q \rightarrow 1$ the Macdonald polynomial $p_{\lambda}$ tends to the corresponding Heckman-Opdam polynomial (with $g_{\alpha}=g, \forall \alpha \in R$ ) [Mac01]. To perform this limit at the level of the above determinantal construction, it suffices to determine the asymptotics of the eigenvalues $\epsilon_{\mu}$ for $q \rightarrow 1$. The asymptotics in question is given by (cf. the proof of Proposition 5.4)

$$
\epsilon_{\mu} t^{-\langle\pi, \rho\rangle}=|W(\pi)|+c_{\pi}\langle\mu+g \rho, \mu+g \rho\rangle(q-1)^{2}+O\left((q-1)^{3}\right),
$$

where $c_{\pi}$ is a positive constant that does not depend on $\mu$ and $g$. Since the formulas of the determinantal construction for the Macdonald polynomials are invariant with respect to an affine rescaling of the spectrum of the form $\epsilon_{\mu} \rightarrow a \epsilon_{\mu}+b$ (with $a \neq 0$ ), we only pick up the second-order term of the asymptotics in Equation (5.7) when sending $q$ to 1 . The upshot is that by replacing $\epsilon_{\mu}$ by $\langle\mu+g \rho, \mu+g \rho\rangle$ in Theorem 5.5, we wind up with an alternative determinantal formula for the Heckman-Opdam polynomials (with $g_{\alpha}=g, \forall \alpha \in R$, and with $R$ such that $R^{\vee}$ has a minuscule weight).

Remark (ii). The recurrence relation in part ii of Theorem 5.5 reads concretely

$$
\left(\epsilon_{\lambda}-\epsilon_{\lambda^{(\ell-1)}}\right) c_{\lambda \lambda^{(\ell-1)}}=\sum_{k=\ell}^{n} \sum_{\kappa \in W\left(\lambda^{(k)}\right) \cap\left(W\left(\lambda^{(\ell-1)}+\rho\right)-\rho\right)} \operatorname{det}\left(w_{\rho+\kappa}\right)\left(\epsilon_{\kappa}-\epsilon_{\lambda}\right) c_{\lambda \lambda^{(k)}} .
$$

This relation should be regarded as a symmetrized Freudenthal type recurrence for the coefficients in the monomial expansion of the Macdonald polynomials. For $\epsilon_{\mu}=\langle\mu+g \rho, \mu+g \rho\rangle$, this recurrence degenerates to a recurrence for the coefficients of the Heckman-Opdam polynomials with $g_{\alpha}=g$, $\forall \alpha \in R$ (cf. Remark (i) above). The recurrence in question is different from the previous recurrence for the Heckman-Opdam polynomials originating from the hypergeometric differential operator (cf. Remark (iii) at the end of $\S 4.2$ ). In particular, for $g=1$ this gives rise to an alternative system of symmetrized Freudenthal type recurrence relations for the weight multiplicities of characters of simple Lie groups.

Remark (iii). For some root systems the minuscule weight $\pi$ is not unique (viz. for $R$ equal to $A_{N}$, $D_{N}$ or $E_{6}$ ). In such a situation, each possible choice for $\pi$ induces a computationally non-equivalent determinantal construction of the corresponding Macdonald polynomials. 


\section{DETERMinANTAL CONSTRUCTION OF POLYNOMIALS}

Example. For $R=D_{3}$ and $\lambda=(2,1,0)$ the determinantal formula reads

$$
\begin{aligned}
& p_{2,1,0}=\left(\left(\epsilon_{2,1,0}^{D}-\epsilon_{1,1,1}^{D}\right)\left(\epsilon_{2,1,0}^{D}-\epsilon_{1,1,-1}^{D}\right)\left(\epsilon_{2,1,0}^{D}-\epsilon_{1,0,0}^{D}\right)\right)^{-1} \\
& \times\left|\begin{array}{cccc}
m_{1,0,0} & \epsilon_{2,1,0}^{D}-\epsilon_{1,0,0}^{D} & 0 & 0 \\
m_{1,1,-1} & -\epsilon_{2,1,0}^{D}+\epsilon_{1,-1,1}^{D} & \epsilon_{2,1,0}^{D}-\epsilon_{1,1,-1}^{D} & 0 \\
m_{1,1,1} & -\epsilon_{2,1,0}^{D}+\epsilon_{1,-1,-1}^{D} & 0 & \epsilon_{2,1,0}^{D}-\epsilon_{1,1,1}^{D} \\
m_{2,1,0} & -\epsilon_{1,-2,0}^{D}+\epsilon_{-1,2,0}^{D} & -2 \epsilon_{2,1,0}^{D}+\epsilon_{1,0,-2}^{D}+\epsilon_{0,2,-1}^{D} & -2 \epsilon_{2,1,0}^{D}+\epsilon_{1,0,2}^{D}+\epsilon_{0,2,1}^{D}
\end{array}\right|,
\end{aligned}
$$

with $\epsilon_{m_{1}, m_{2}, m_{3}}^{D}=\left(t^{4} q^{m_{1}}+q^{-m_{1}}\right)+\left(t^{3} q^{m_{2}}+t q^{-m_{2}}\right)+\left(t^{2} q^{m_{3}}+t^{2} q^{-m_{3}}\right)$ or with $\epsilon_{m_{1}, m_{2}, m_{3}}^{D}=$ $q^{-\left(m_{1}+m_{2}+m_{3}\right) / 2}\left(t^{2} q^{m_{1}}+1\right)\left(t q^{m_{2}}+1\right)\left(q^{m_{3}}+1\right)$ (depending on the choice for the minuscule weight).

\section{Macdonald polynomials: the case of general $t_{\alpha}$}

In this section we will briefly indicate how to generalize the results of the previous section to the case of Macdonald polynomials with general parameters $t_{\alpha}$ such that $t_{w(\alpha)}=t_{\alpha}$ for all $w \in W$. We will keep the restriction that our root system $R$ is reduced and that the dual root system $R^{\vee}$ has a minuscule weight $\pi$.

For general $W$-invariant $t_{\alpha}$-parameters the Macdonald operator becomes [Mac01]

$$
D_{\pi}=\frac{1}{\left|W_{\pi}\right|} \sum_{w \in W}\left(\prod_{\alpha \in R^{+}} \frac{1-t_{\alpha}^{\langle\pi, \alpha\rangle} e^{w(\alpha)}}{1-e^{w(\alpha)}}\right) T_{w(\pi), q} .
$$

It is convenient to reparameterize the $t_{\alpha}$ as

$$
t_{\alpha}=q^{g_{\alpha}}
$$

(with $g_{w(\alpha)}=g_{\alpha}, \forall w \in W$ ). The action of $D_{\pi}$ on the monomial basis can be written as [Mac01, Section 5]

$$
D_{\pi} m_{\lambda}=q^{\left\langle\pi, \rho_{g}\right\rangle} \sum_{X \subset R^{+}}(-1)^{|X|} \sum_{\nu \in W(\lambda)} q^{\left\langle\pi, \nu+\rho_{g}\left(X^{c}\right)-\rho_{g}(X)\right\rangle} \chi_{\nu-2 \rho(X)},
$$

where $X^{c}=R^{+} \backslash X$, and

$$
\rho(X)=\frac{1}{2} \sum_{\alpha \in X} \alpha, \quad \rho_{g}(X)=\frac{1}{2} \sum_{\alpha \in X} g_{\alpha} \alpha
$$

(so $\rho=\rho\left(R^{+}\right)$and $\rho_{g}=\rho_{g}\left(R^{+}\right)$). Bringing the action in Equation (6.2) to triangular form gives

$$
D_{\pi} m_{\lambda}=\epsilon_{\lambda} \chi_{\lambda}+\sum_{\mu \in \mathcal{P}^{+}, \mu \prec \lambda} b_{\lambda \mu} \chi_{\mu}
$$

with

$$
\begin{aligned}
& \epsilon_{\lambda}=q^{\left\langle\pi, \rho_{g}\right\rangle} \sum_{\tau \in W(\pi)} q^{\left\langle\tau, \lambda+\rho_{g}\right\rangle},
\end{aligned}
$$

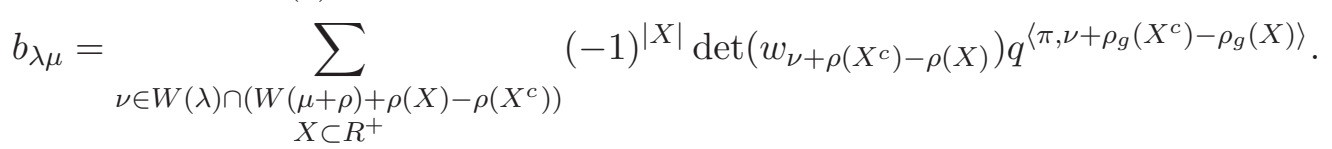

We thus wind up with the following determinantal construction of the Macdonald polynomials for general $W$-invariant parameters. 


\section{J. F. van Diejen, L. Lapointe And J. Morse}

Theorem 6.1 (Determinantal construction). For $\lambda \in \mathcal{P}^{+}$, let

$$
p_{\lambda}=m_{\lambda}+\sum_{\mu \in \mathcal{P}^{+}, \mu \prec \lambda} c_{\lambda \mu} m_{\mu}
$$

denote the (monic) Macdonald polynomial with $t_{\alpha}=q^{g_{\alpha}}$. Then, upon setting for $\mu, \nu \in \mathcal{P}^{+}$

$$
\begin{aligned}
\epsilon_{\mu} & =q^{\left\langle\pi, \rho_{g}\right\rangle} \sum_{\tau \in W(\pi)} q^{\left\langle\tau, \mu+\rho_{g}\right\rangle}, \\
d_{\mu \nu} & =b_{\mu \nu}-\epsilon_{\lambda} a_{\mu \nu},
\end{aligned}
$$

with

$$
\begin{aligned}
a_{\mu \nu} & =\sum_{\kappa \in W(\mu) \cap(W(\nu+\rho)-\rho)} \operatorname{det}\left(w_{\rho+\kappa}\right), \\
b_{\mu \nu} & \sum_{\kappa \in W(\mu) \cap\left(W(\nu+\rho)+\rho(X)-\rho\left(X^{c}\right)\right)}(-1)^{|X|} \operatorname{det}\left(w_{\kappa+\rho\left(X^{c}\right)-\rho(X)}\right) q^{\left\langle\pi, \kappa+\rho_{g}\left(X^{c}\right)-\rho_{g}(X)\right\rangle} \\
&
\end{aligned}
$$

(so $\left.d_{\mu \mu}=\epsilon_{\mu}-\epsilon_{\lambda}\right)$, we have that:

i) the polynomial $p_{\lambda}$ is represented explicitly by the determinantal formula in Theorem 3.1;

ii) the coefficients $c_{\lambda \mu}$ of its monomial expansion are generated by the linear recurrence in Corollary 3.2;

iii) the expansion coefficients $c_{\lambda \mu}$ are given in closed form by the formula in Corollary 3.3.

Remark (i). From a computational standpoint the formulas of Theorem 6.1 are much less effective than the determinantal constructions for the $(q, t)$ Macdonald polynomials (Theorem 5.5) and (especially) for the Heckman-Opdam polynomials (Theorem 4.4). This is because the action of the general Macdonald operator on the monomial basis (cf. Equation (6.2)) is much more complex than in these two previous cases. This renders Theorem 6.1 presumably only of limited practical value.

Remark (ii). The most general class of Macdonald polynomials admits a richer parameter structure connected with admissible pairs of root systems $(R, S)$ [Mac01]. (From this perspective the polynomials studied here are of the type $(R, R)$.) Since Macdonald in fact gives the action of the Macdonald operator on the monomial basis for general admissible pairs, it is not difficult to generalize Theorem 6.1 also to this context (at the expense of having to introduce a more elaborate notational apparatus).

\section{REFERENCES}

Bou68 N. Bourbaki, Groupes et algèbres de Lie, (Hermann, Paris, 1968), chaps. 4-6.

Die96 J. F. van Diejen, On the diagonalization of difference Calogero-Sutherland systems, in Symmetries and integrability of difference equations, eds D. Levi, L. Vinet and P. Winternitz, CRM Proc. Lecture Notes, vol. 9 (American Mathematical Society, Providence, RI, 1996), 79-89.

HS94 G. Heckman and H. Schlichtkrull, Harmonic analysis and special functions on symmetric spaces, Perspect. Math., vol. 16 (Academic Press, San Diego, 1994).

Hel94 S. Helgason, Geometric analysis on symmetric spaces, Mathematical Surveys and Monographs, vol. 39 (American Mathematical Society, Providence, RI, 1994).

Hum72 J. E. Humphreys, Introduction to Lie algebras and representation theory (Springer-Verlag, New York, 1972).

LLM98 L. Lapointe, A. Lascoux and J. Morse, Determinantal expressions for Macdonald polynomials, Int. Math. Res. Notices (1998), 957-978. 


\section{Determinantal CONSTRUCTION OF POLYNOMials}

LLM00 L. Lapointe, A. Lascoux and J. Morse, Determinantal expression and recursion for Jack polynomials, Electron. J. Combin. 7 (2000), Note 1, 7 pp. (electronic).

Mac95 I. G. Macdonald, Symmetric functions and Hall polynomials, 2nd edn (Oxford University Press, New York, 1995).

Mac98 I. G. Macdonald, Symmetric functions and orthogonal polynomials, University Lecture Series, vol. 12 (American Mathematical Society, Providence, RI, 1998).

Mac01 I. G. Macdonald, Orthogonal polynomials associated with root systems, Sém. Lothar. Combin. 45 (2001), Art. B45a, 40 pp. (electronic).

MP82 R. V. Moody and J. Patera, Fast recursion formula for weight multiplicities, Bull. Amer. Math. Soc. (N.S.) 7 (1982), 237-242.

Nou96 M. Noumi, Macdonald's symmetric polynomials as zonal spherical functions on some quantum homogeneous spaces, Adv. Math. 123 (1996), 16-77.

OP83 M. A. Olshanetsky and A. M. Perelomov, Quantum integrable systems related to Lie algebras, Phys. Rep. 94 (1983), 313-404.

Opd95 E. M. Opdam, Harmonic analysis for certain representations of graded Hecke algebras, Acta Math. 175 (1995), 75-121.

Rui87 S. N. M. Ruijsenaars, Complete integrability of relativistic Calogero-Moser systems and elliptic function identities, Comm. Math. Phys. 110 (1987), 191-213.

Sta89 R. P. Stanley, Some combinatorial properties of Jack symmetric functions, Adv. Math. 77 (1989), 76-115.

Sug99 T. Sugitani, Zonal spherical functions on quantum Grassmann manifolds, J. Math. Sci. Univ. Tokyo 6 (1999), 335-369.

Sut71 B. Sutherland, Exact results for a quantum many-body system in one dimension, I, Phys. Rev. A4 (1971), 2019-2021

Sut72 B. Sutherland, Exact results for a quantum many-body system in one dimension, II, Phys. Rev. A5 (1972), 1372-1376.

Wil88 J. H. Wilkinson, The algebraic eigenvalue problem (Oxford University Press, New York, 1988).

J. F. van Diejen diejen@inst-mat.utalca.cl

Instituto de Matemática y Física, Universidad de Talca, Casilla 747, Talca, Chile

L. Lapointe lapointe@inst-mat.utalca.cl

Instituto de Matemática y Física, Universidad de Talca, Casilla 747, Talca, Chile

J. Morse morsej@math.miami.edu

Department of Mathematics, University of Miami, Coral Gables, FL 33124, USA 